Scientific Paper

\title{
Dose calculation accuracy for photon small fields in treatment planning systems with comparison by Monte Carlo simulations
}

\author{
Mojtaba ABAZARFARDa ${ }^{\mathrm{a}}$ Payam AZADEH ${ }^{\mathrm{b}}$, Ahmad MostAAR ${ }^{\mathrm{a}^{*}}$ \\ ${ }^{a}$ Department of Medical Physics and Biomedical Engineering, School of Medicine, Shahid Beheshti University of Medical \\ Sciences, Tehran, Iran \\ ${ }^{b}$ Department of Radiation Oncology, School of Medicine, Shahid Beheshti University of Medical Sciences, Tehran, Iran \\ *E-mail address: mostaar@sbmu.ac.ir
}

\begin{abstract}
Purpose: Advanced radiation therapy techniques use small fields in treatment planning and delivery. Small fields have the advantage of more accurate dose delivery, but with the cost of some complications in dosimetry. Different dose calculation algorithms imported in various treatment planning systems (TPSs) which each of them has different accuracy. Monte Carlo (MC) simulation has been reported as one of the accurate methods for calculating dose distribution in radiation therapy. The aim of this study was the evaluation of TPS dose calculation algorithms in small fields against 2 MC codes.

Methods: A linac head was simulated in $2 \mathrm{MC}$ codes, MCNPX, and GATE. Then three small fields $(0.5 \times 0.5,1 \times 1$ and $1.5 \times 1.5 \mathrm{~cm}^{2}$ ) were simulated with $2 \mathrm{MC}$ codes, and also these fields were planned with different dose calculation algorithms in Isogray and Monaco TPS. PDDs and lateral dose profiles were extracted and compared between MC simulations and dose calculation algorithms.

Results: For $0.5 \times 0.5 \mathrm{~cm}^{2}$ field mean differences in PDDs with MCNPX were 2.28, 4.6, 5.3, and 7.4\% and with GATE were $-0.29,2.3,3$ and $5 \%$ for CCC, superposition, FFT and Clarkson algorithms respectively. For $1 \times 1 \mathrm{~cm}^{2}$ field mean differences in PDDs with MCNPX were 1.58, 0.6, 1.1 and 1.4\% and with GATE were 0.77, 0.1, 0.6 and 0.9\% for CCC, superposition, FFT and Clarkson algorithms respectively. For $1.5 \times 1.5 \mathrm{~cm}^{2}$ field mean differences in PDDs with MCNPX were $0.82,0.4,0.6$ and $-0.4 \%$ and with GATE were $2.38,2.5,2.7$ and $1.7 \%$ for CCC, superposition, FFT and Clarkson algorithms respectively.

Conclusions: Different dose calculation algorithms were evaluated and compared with MC simulation in small fields. Mean differences with MC simulation decreased with the increase of field sizes for all algorithms.
\end{abstract}

Key words: small field; radiation therapy; Monte Carlo simulation; MCNPX; GATE; dose calculation algorithms.

\section{Introduction}

Advanced radiation therapy techniques such as IMRT, SBRT, and VMAT are using small photon beamlets (smaller than $4 \times 4$ $\mathrm{cm}^{2}$ ) for treatment planning and treatment delivery. ${ }^{\mathbf{1 - 3}}$ Steep dose gradient in these fields results in a higher dose to target volume and simultaneously less dose to organs at risk (OARs), ${ }^{4-6}$ this is the ultimate goal in radiation therapy. ${ }^{5}$ However, using small fields will make some trouble in beam dosimetry. ${ }^{1}$ The partial occlusion of primary photon beam from source, loss of lateral charged particle equilibrium, steep dose gradient and volume averaging in dosimeter are the major issues that make dosimetry in the small field a challenging work. ${ }^{1,2,7-9}$ Because of these problems, there is no ideal dosimeter for use in small fields. ${ }^{9}$ Tissue equivalent, energy and dose rate independence, linear response, high resolution and small relative size are the ideal characteristics of a suitable detector for the dosimetry of small fields. ${ }^{1}$ Various dosimeters have been used for small field dosimetry despite all challenges and limitations, such as small volume ion chambers, radiochromic films, diodes, diamond detectors, and plastic scintillators. ${ }^{\mathbf{1 , 1 0 , 1 1}}$

Monte Carlo (MC) simulation was used to calculate dose distribution in small field radiotherapy, in various studies., 2,12-14 Since MC simulation takes the exact geometry of linac, electron transport, and beam configurations into account, ${ }^{15-16}$ it precision does not limit by complications from advanced techniques (such as IMRT and small fields) and tissue heterogeneities. ${ }^{\mathbf{1 5}}$ It was mentioned that MC simulation is one of the most accurate approaches to calculate dose distribution in radiation therapy dosimetry. ${ }^{1,2,12,15-17}$ MC techniques are accepted as the gold standard in radiotherapy dose calculation. ${ }^{\mathbf{1 , 1 8}}$ It is known that MC will give accurate dose calculation in regions of charged particle disequilibrium and where measurement interpretation is challenging. ${ }^{1}$ So MC simulation has considered as standard practice in benchmarking dose calculation algorithms used in

(C) 2021 Mojtaba Abazarfard, Payam Azadeh, Ahmad Mostaar. This is an open access article licensed under the Creative Commons Attribution-NonCommercialNoDerivs License (http://creativecommons.org/licenses/by-nc-nd/4.0/). 
commercial treatment planning systems. ${ }^{\mathbf{1 , 6 , 1 2}}$ However MC simulation accuracy is limited by time of calculation, ${ }^{\mathbf{1 9}}$ and this is why these algorithms aren't used widely in commercial treatment planning systems. ${ }^{\mathbf{2 0}}$ There are several different MC codes available for simulating a radiation therapy unit, including EGSnrc, ETRAN, Geant4, and MCNPX. ${ }^{21}$ MCNPX is widely used to simulate medical linear accelerators employed in radiotherapy. 2,22 GATE (Geant4 Application for Emission Tomography) is a Monte Carlo platform that helped neuromas studies in nuclear medicine. A recent version of this code enabled the modeling of X-ray computed tomography and radiotherapy. ${ }^{23}$ Sarrut et al demonstrated the potential and versatility of this code for dosimetry and radiotherapy applications since it's open-source and user-friendly. ${ }^{\mathbf{2 4}}$ It is expected that GATE will have an increasing role in combined therapy and imaging.

The maximum error in dose delivery in the entire radiotherapy process is desired to be less than $5 \%,{ }^{\mathbf{1 6 , 1 8 , 2 5 , 2 6}}$ therefore it needs all steps of this process to be in an optimal situation to decrease the error as much as possible. Treatment planning is an important step in this process and the dose calculation algorithm is the most important part of it, which determines the accuracy of the treatment planning system. There are two types of dose calculation algorithms: measurement-based (such as pencil beam convolution (PBC) algorithm) and model-based (such as anisotropic analytical algorithm (AAA), collapsed cone convolution (CCC) and Acuros XB (AXB) algorithm). ${ }^{\mathbf{1} 6}$ Small field dosimetry issues are the origin of inaccuracies in dose calculation algorithms since measurement data is required in algorithm configuration., ${ }^{\mathbf{6} 27}$ This problem has notable outcomes in the accuracy of measurement-based algorithms. But in model-based algorithms inherent performance of the algorithm determines the accuracy of dose calculation. ${ }^{6}$ These inaccuracies are mostly due to the inability of the algorithm to model the loss of electronic equilibrium, ${ }^{\mathbf{6}, 15,16}$ which are specific to measurement-based algorithms that are usually based on pencil beam convolution. ${ }^{28}$ More advanced algorithms, modelbased, which are based on superposition convolution principles, have more acceptable results since they model the loss of charged particle disequilibrium. ${ }^{28}$ It was shown that PBC algorithm (measurement-based) overestimate dose to lung inhomogeneities and small fields (up to $30 \%$ in some cases) where the loss of lateral charged particle equilibrium happens. ${ }^{\mathbf{1 6 , 1 8 , 2 9 , 3 0}}$ However model-based algorithms such as AAA and CCC have reliable and more accurate dose calculations. 1,15,27,30,31 There is an Acuros XB algorithm that solves the linear Boltzmann transport equation which describes the macroscopic conduct of radiation particles throughout the matter. ${ }^{32}$ Several studies have compared the AAA algorithm with AXB and MC simulation., ${ }^{\mathbf{1 , 3 2}}$ Results showed that AXB has superior accuracy in comparison with AAA in the region of electronic disequilibrium and tissue heterogeneities. ${ }^{31}$ These differences are reported to be due to beam energy, field sizes and density of materials. ${ }^{33}$ Fogliata and Cozzi made a review of different types of dose calculation algorithms that are being used in SBRT TPS for lung cancer cases. ${ }^{28}$ They concluded that measurement-based algorithms aren't suitable for such cases (small fields and low density) which they show differences in the order of $20-30 \%$, while model-based and more advanced (AXB) algorithms provide more accurate results and have differences in the order of $10 \%$ and $5 \%$ respectively.

The aim of the current study was to evaluate the performance of four dose calculation algorithms: collapsed cone convolution (CCC) employed in Monaco treatment planning system, superposition-convolution, fast Fourier transform (FFT) and Clarkson used in Isogray TPS in comparison with Monte Carlo simulation in small photon fields in a homogeneous water phantom. We employed 2 MC codes, MCNPX and GATE.

\section{Materials and methods}

\section{MC simulation}

MC simulations are a type of mathematical method for calculating dose distribution by transporting electrons and photons based on principles of radiation transport. In this study, we used two different MC codes. Models for both codes are described below.

\section{A. $M C N P X$}

MCNPX Monte Carlo code ${ }^{34}$ was used to simulate Elekta Synergy Platform linear accelerator. Linacs have a modular construction that is specified by different vendors. The vendor's specified data was used to simulate all necessary parts of linac's head, including electron target, primary collimator, flattening filter, ionization chambers, mirror, and secondary definition jaws. The model also consisted of a $40 \times 40 \times 40 \mathrm{~cm}^{3}$ cylindrical water phantom. $6 \mathrm{MeV}$ photon beam was used for simulation. This model was divided into two parts: 1) patient independent parts (including electron target, primary collimator, flattening filter, ionization chambers and mirror), and 2) patient dependent part (including secondary collimators and water phantom). By scoring photons that cross a plane above secondary collimators a phase space file was generated (represent patient independent parts). Then the phase space was used as a photon point source at $100 \mathrm{~cm}$ source to surface distance (SSD). For different calculation purposes, different field sizes were required which were designed by changing the opening of secondary collimators. Initial electron energy was benchmarked by comparing the measured and calculated data.

For model verification, we simulated 3 larger fields $(6 \times 6$, $10 \times 10$ and $20 \times 20 \mathrm{~cm}^{2}$ ), where challenges due to small field complexity do not exist. ${ }^{2}$ For these fields, PDDs and beam profiles were calculated and compared with direct measurement data. Direct measurements were done by a $0.125 \mathrm{~cm}^{3}$ Semiflex ion chamber (type 31010, PTW-Freiburg, Germany) in a MP3 water phantom (PTW-Freiburg, Germany). 
For comparison with treatment planning system outputs, three small fields $\left(0.5 \times 0.5,1 \times 1\right.$ and $\left.1.5 \times 1.5 \mathrm{~cm}^{2}\right)$ were simulated. To calculate PDDs in the water phantom, a set of cylindrical scoring cells with a radius equal to $10 \%$ of the beam's diameter ${ }^{35}$ and 2 $\mathrm{mm}$ height was used along the beam's central axis. For profile calculation, a set of scoring cells with $2 \times 3 \times 3 \mathrm{~mm}^{3}$ dimensions was used in the depth of maximum dose and $10 \mathrm{~cm}$ across the central axis. By running particle histories through the water phantom, deposited energy in scoring cells was calculated by *F8 tally. Electron and photon energy cut-off was set to 0.4 and $0.01 \mathrm{MeV}$ respectively. Calculations were done on a workstation, by running about $2 \times 10^{9}$ histories for each field size, the statistical uncertainty was less than $0.5 \%$ for all of the calculations.

\section{B. GATE simulation}

GATE is an advanced open-source software package developed by OpenGATE collaboration dedicated to numerical simulation in medical imaging and radiation therapy. This code is userfriendly and uses command scripts. GATE is based on Geant4 and uses its libraries. The radiation therapy option is added to this code in version 6.2. This code considers all physical phenomena and the user can determine which physical phenomenon to be considered in the physics list input file. Emstandard_OPT3 physics list from Geant4 was used in our model. In this study, version 8.2 was used to simulate Elekta Synergy Platform linear accelerator. Geometry data was provided by the vendor. All necessary parts were simulated. The model included an electron target, primary collimator, flattening filter, ionization chambers, mirror, secondary collimators, and a $40 \times 40 \times 40 \mathrm{~cm}^{3}$ homogeneous water phantom. Different field sizes were simulated for further calculation. The initial electron energy was $6 \mathrm{MeV}$. For energy benchmarking, direct measurement data was used, this data was the same as those were used for MCNPX benchmarking. Model verification was carried out similarly to the MCNPX model.

In GATE just like simulation in MCNPX, simulation was divided into two parts, patient independent part and patient dependent part. Like MCNPX a phase space file was generated by scoring the photons that crossing a plane above secondary collimators. Then the phase space file was used as a point source at $100 \mathrm{~cm}$ source to surface distance. Same as MCNPX, three small fields $\left(0.5 \times 0.5,1 \times 1\right.$ and $\left.1.5 \times 1.5 \mathrm{~cm}^{2}\right)$ were simulated. Cut-offs were set to $2 \mathrm{~mm}$ for photons and $1 \mathrm{~mm}$ for electrons, which are equal to 10 and $350 \mathrm{keV}$ respectively. Calculating dose distribution was done by attaching an actor to the water phantom. This actor is Dose Actor. The Dose Actor output file is a $3 \mathrm{D}$ matrix in mhd or raw formats. The voxel size on this matrix was $2 \times 2 \times 2 \mathrm{~mm}^{3}$. This actor has different output data, including dose, dose uncertainty, energy deposited, energy deposited uncertainty, etc. Users can define which one of these output data's to be active or not. For this work dose and dose uncertainty was needed. Then dose distribution file was imported into MATLAB software and PDDs and profiles were extracted. After running about $2 \times 10^{9}$ particle histories, uncertainty in all fields was less than $2 \%$.

\section{TPS data acquisition}

\section{A. Dose calculation in Isogray TPS}

Isogray version 4.1.3 treatment planning system (Dosisoft Co., France) was used in this study. This TPS has different dose calculation algorithms including superposition convolution, fast Fourier transform, and Clarkson. This TPS works with various techniques such as IMRT. Algorithm commissioning was carried out precisely using the vendor's instructions and recommendations. For this study superposition convolution, fast Fourier transform and Clarkson algorithms were used. The First 2 of these algorithms are model-based and the latest is measurement-based. Beam configurations were done for three small fields, $0.5 \times 0.5,1 \times 1$ and $1.5 \times 1.5 \mathrm{~cm}^{2}$, where source to surface distance was $100 \mathrm{~cm}$. dose distribution calculation was done in a $40 \times 40 \times 40 \mathrm{~cm}^{3}$ homogeneous water phantom created in the treatment planning system. For all plans, $6 \mathrm{MeV}$ beam energy was used. Dose calculation grid size was set to the minimum size available, which was $2 \times 2 \times 2 \mathrm{~mm}^{3}$. All plans calculated dose distribution using all algorithms. To investigate calculated dose distribution, PDDs and profiles were extracted. PDDs extracted along the beam's central axis and profiles in 2 depths, depth of maximum dose, and $10 \mathrm{~cm}$.

\section{B. Dose calculation in Monaco TPS}

Collapsed cone convolution (CCC) dose calculation algorithm implemented in Monaco 5.11.02 was also evaluated in the current study. This algorithm was commissioned by the manufactory's recommendation. All previous plans for different field sizes from Isogray were repeated in Monaco with the CCC dose calculation algorithm and with the dose calculation grid size of $2 \times 2 \times 2 \mathrm{~mm}^{3}$.

\section{Results}

\section{Validation of Monte Carlo simulation}

A new formalism and machine-specific reference field for a situation that cannot establish conventionally were introduced by Alfonso et al. for calibration intentions. ${ }^{3}$ In this study, a standard reference field $10 \times 10$, also $20 \times 20$ and $6 \times 6 \mathrm{~cm}^{2}$ were used as machine-specific reference fields for model validation. $6 \times 6 \mathrm{~cm}^{2}$ field was used because its closer to small fields and charged particle equilibrium still exists. Primary electron source energy was determined by trial and error by comparing PDD and Profiles (in the depth of $10 \mathrm{~cm}$ ) from simulation and measurement in a homogeneous water phantom and $10 \times 10 \mathrm{~cm}^{2}$ field size. Figure 1 shows a comparison in PDDs between $2 \mathrm{MC}$ simulations (MCNPX and GATE) and measurement for $6 \times 6$, $10 \times 10$, and $20 \times 20 \mathrm{~cm}^{2}$ fields. For the $6 \times 6 \mathrm{~cm}^{2}$ field, the mean differences between MCNPX and GATE with measurement were $0.59 \%$ and $-0.2 \%$ respectively with the maximum difference $(-14 \%$ and $7 \%$ respectively) being in the buildup 
region. For the $10 \times 10 \mathrm{~cm}^{2}$ field, the mean differences were $0.39 \%$ and $-0.5 \%$ respectively and for the $20 \times 20 \mathrm{~cm}^{2}$ field, the mean differences were $-2.4 \%$ and $-1.9 \%$ respectively. Figure 2 shows beam profiles for $20 \times 20,10 \times 10$, and $6 \times 6 \mathrm{~cm}^{2}$ Fields. Statistical uncertainty for both MC codes was below $0.5 \%$. Venselaar et al. divided dose profiles into three regions: 1) the umbra or flat region inside the field, 2) the penumbra region and 3 ) the low dose region outside the field. ${ }^{36}$ There are several recommendations for evaluating calculations in different situations, one of them introduced by Venselaar et al. where recommend criteria for acceptance of calculation as follow, for 1) umbra region $2 \%$ difference, 2 ) for penumbra region $10 \%$ and for 3) low dose region $30 \%{ }^{36}$ For the $6 \times 6 \mathrm{~cm}^{2}$ field, there were the mean difference of $-0.36 \%$ and $0.009 \%$ inside the field for MCNPX and GATE respectively. For the penumbra region, these differences were $-9.6 \%$ for MCNPX and $-10.4 \%$ for GATE. And finally, for the low dose region mean differences

(a)

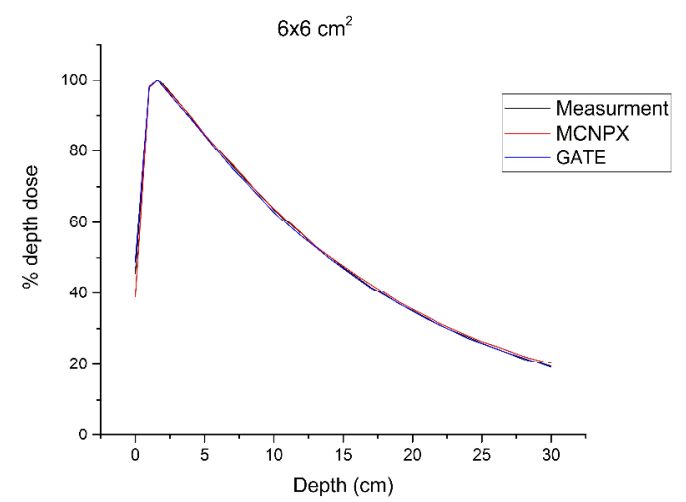

(b)

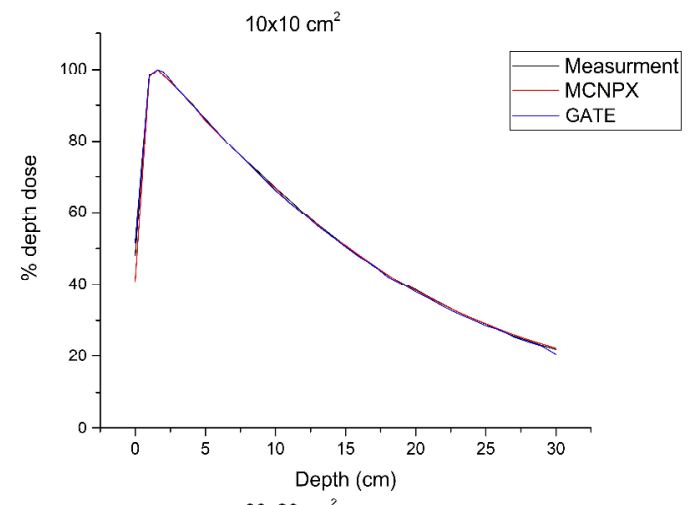

(c)

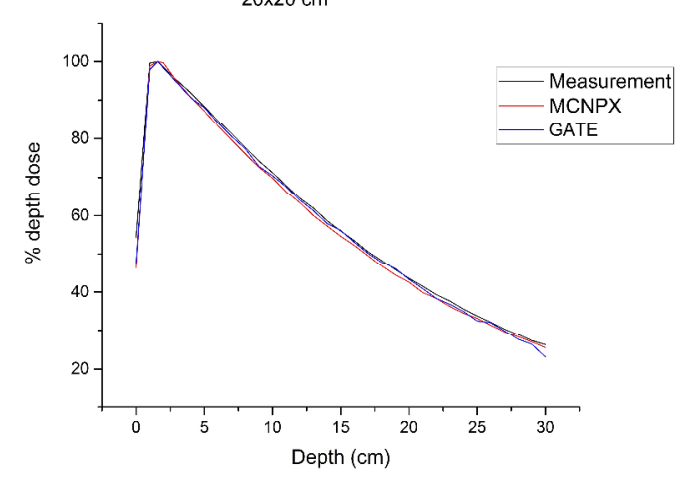

Figure 1. PDD curves in a homogenous water phantom, a comparison between measurements, MCNPX and GATE for various field sizes. A) $6 \times 6 \mathrm{~cm}^{2}$ and B) $10 \times 10 \mathrm{~cm}^{2}$. C) $20 \times 20 \mathrm{~cm}^{2}$. were $-28.8 \%$ and $-18.2 \%$ for MCNPX and GATE. For $10 \times 10$ $\mathrm{cm}^{2}$ field, the mean differences were $-1 \%$ and $-0.6 \%$ for flat region, $-2.5 \%$ and $-9.8 \%$ for penumbra region and $-28.7 \%$ and $19.5 \%$ for low dose region for MCNPX and GATE respectively and for $20 \times 20 \mathrm{~cm}^{2}$ field, the mean differences were $-0.3 \%$ and $0.4 \%$ for flat region, $-17.6 \%$ and $-17.7 \%$ for penumbra region and $-28.4 \%$ and $-28.7 \%$ for low dose region for MCNPX and GATE respectively. For the $6 \times 6 \mathrm{~cm}^{2}$ field, full width at half maximum (FWHM) was 6.75, 6.68 and $6.62 \mathrm{~cm}$ for measurement, MCNPX and GATE respectively. For the $10 \times 10 \mathrm{~cm}^{2}$ field, FWHM was 11.06, 11.09, and $11 \mathrm{~cm}$ for measurement, MCNPX, and GATE respectively. For the $20 \times 20$ $\mathrm{cm}^{2}$ field, FWHM was 23.89, 23.91, and $23.92 \mathrm{~cm}$ for measurement, MCNPX, and GATE respectively. Differences in FWHM were less than $2 \mathrm{~mm}$ between measurement and calculations for all fields.

(a)

(b)

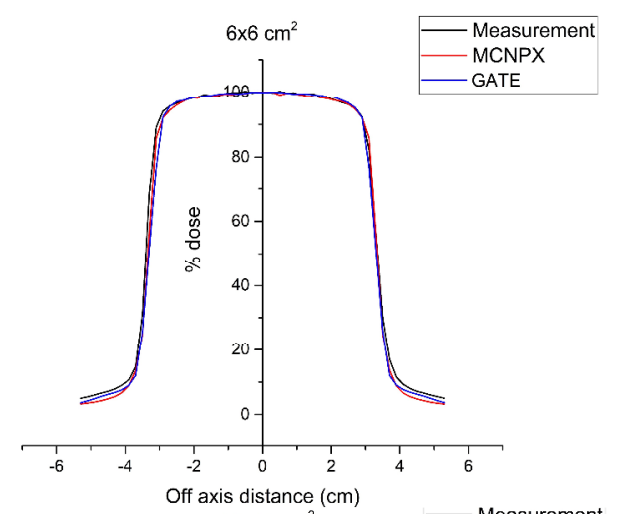

)

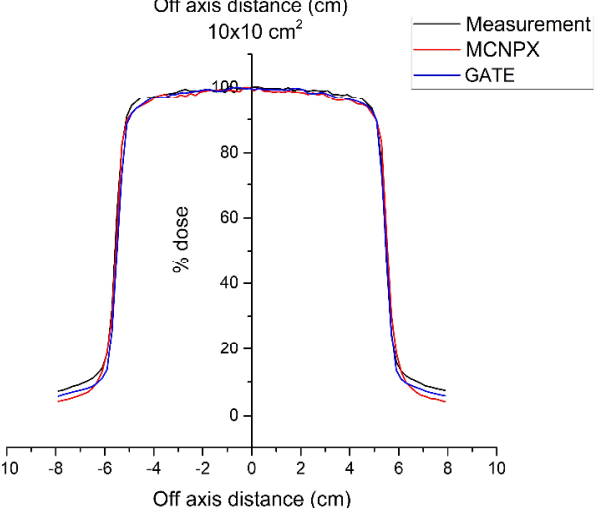

(c)

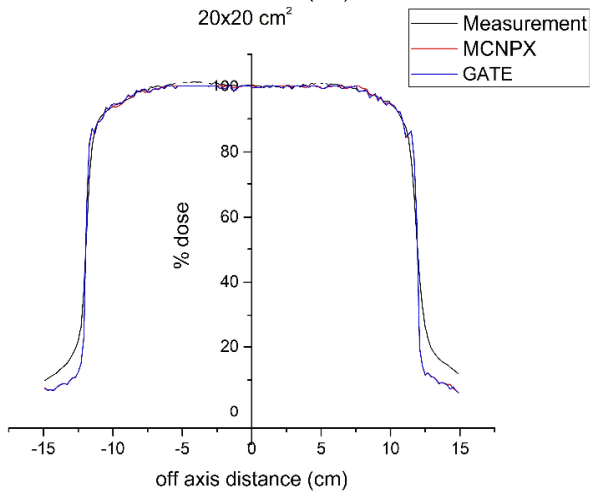

Figure 2. Lateral beam profiles in a homogenous water phantom, a comparison between measurements, MCNPX and GATE for various field sizes. A) $6 \times 6 \mathrm{~cm}^{2}$ and B) $10 \times 10 \mathrm{~cm}^{2}$. C) $20 \times 20 \mathrm{~cm}^{2}$. 


\section{Comparing PDDs between TPSs and MCNPX and Gate in a small field}

In this study, three small fields were investigated between 2 TPSs and Monte Carlo simulations, $0.5 \times 0.5,1 \times 1$, and $1.5 \times 1.5$ $\mathrm{cm}^{2}$. Calculations for both Monte Carlo codes were done for $2 \times 10^{9}$ histories and statistical uncertainty was below $1 \%$ for all situations and fields. Figure 3 shows a comparison between PDDs for dose calculation algorithms and MCNPX and GATE for different field sizes. Four dose calculation algorithms were evaluated in the current study, CCC, superposition, FFT and Clarkson. The First three of these algorithms are model-based and the latest is correction-based. For $0.5 \times 0.5 \mathrm{~cm}^{2}$ field mean

(a)



(b)

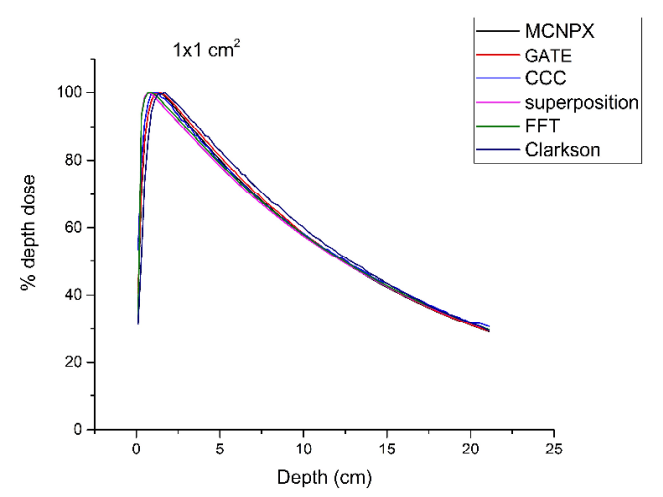

(c)

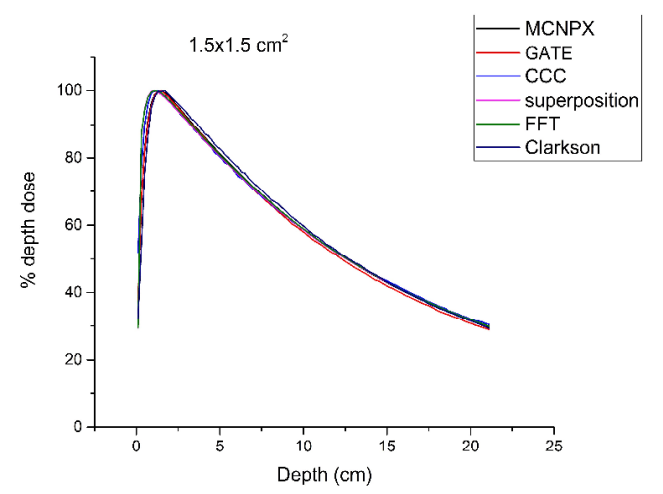

Figure 3. PDD curves for small fields in a homogenous water phantom, a comparison between MCNPX, GATE and various dose calculation algorithms in different field sizes. A) $0.5 \times 0.5 \mathrm{~cm}^{2}$, B) $1 \times 1 \mathrm{~cm}^{2}$ and C) $1.5 \times 1.5 \mathrm{~cm}^{2}$. differences in PDDs with MCNPX were $2.28 \%, 4.6 \%, 5.3 \%$ and $7.4 \%$ and with GATE were $-0.29 \%, 2.3 \%, 3 \%$ and $5 \%$ for CCC, superposition, FFT and Clarkson algorithms respectively. For $1 \times 1 \mathrm{~cm}^{2}$ field, the mean differences in PDDs with MCNPX were $1.58 \%, 0.6 \%, 1.1 \%$ and $1.4 \%$ and with GATE were $0.77 \%$, $0.1 \%, 0.6 \%$ and $0.9 \%$ for CCC, superposition, FFT and Clarkson algorithms respectively. For $1.5 \times 1.5 \mathrm{~cm}^{2}$ field, the mean differences in PDDs with MCNPX were $0.82 \%, 0.4 \%, 0.6 \%$ and $-0.4 \%$ and with GATE were $2.38 \%, 2.5 \%, 2.7 \%$ and $1.7 \%$ for CCC, superposition, FFT and Clarkson algorithms respectively. Relative depth doses for $0.5 \times 0.5,1 \times 1,1.5 \times 1.5 \mathrm{~cm}^{2}$ field are shown in Tables 1, 2 and $\mathbf{3}$ respectively.

Table 1. Relative depth doses (percentage) calculated by different dose calculation algorithms and 2 Monte Carlo codes (MCNPX and GATE) for $0.5 \times 0.5 \mathrm{~cm}^{2}$ field size.

\begin{tabular}{ccccccc}
\hline \hline $\begin{array}{c}\text { Depth } \\
(\mathbf{c m})\end{array}$ & $\begin{array}{c}\text { MCNPX } \\
(\boldsymbol{\%})\end{array}$ & $\begin{array}{c}\text { GATE } \\
(\boldsymbol{\%})\end{array}$ & $\begin{array}{c}\text { CCC } \\
(\boldsymbol{\%})\end{array}$ & $\begin{array}{c}\text { Superposition } \\
(\boldsymbol{\%})\end{array}$ & $\begin{array}{c}\text { FFT } \\
(\boldsymbol{\%})\end{array}$ & $\begin{array}{c}\text { Clarkson } \\
(\boldsymbol{\%})\end{array}$ \\
\hline $\mathbf{0}$ & 46.36 & 45.43 & 32.14 & 32.4 & 32 & 30.37 \\
$\mathbf{1 . 1}$ & 100 & 100 & 99.68 & 97.74 & 98.67 & 97.29 \\
$\mathbf{5}$ & 77.69 & 80.23 & 78.99 & 77.3 & 78.27 & 83.31 \\
$\mathbf{1 0}$ & 55.92 & 57.58 & 57.41 & 57.37 & 57.87 & 60.92 \\
$\mathbf{1 5}$ & 40.69 & 41.79 & 42.61 & 42.76 & 43.07 & 44.64 \\
$\mathbf{2 0}$ & 29.94 & 30.69 & 31.36 & 32.14 & 32.27 & 32.97 \\
\hline \hline
\end{tabular}

Table 2. Relative depth doses (percentage) calculated by different dose calculation algorithms and 2 Monte Carlo codes (MCNPX and GATE) for $1 \times 1 \mathrm{~cm}^{2}$ field size.

\begin{tabular}{ccccccc}
\hline $\begin{array}{c}\text { Depth } \\
(\mathbf{c m})\end{array}$ & $\begin{array}{c}\text { MCNPX } \\
(\boldsymbol{\%})\end{array}$ & $\begin{array}{c}\text { GATE } \\
(\boldsymbol{\%})\end{array}$ & $\begin{array}{c}\text { CCC } \\
(\boldsymbol{\%})\end{array}$ & $\begin{array}{c}\text { Superposition } \\
(\boldsymbol{\%})\end{array}$ & $\begin{array}{c}\text { FFT } \\
(\boldsymbol{\%})\end{array}$ & $\begin{array}{c}\text { Clarkson } \\
(\boldsymbol{\%})\end{array}$ \\
\hline $\mathbf{0}$ & 39.61 & 40.59 & 53.52 & 31.81 & 31.51 & 31.28 \\
$\mathbf{1 . 3}$ & 100 & 100 & 100 & 97.54 & 98.55 & 99.57 \\
$\mathbf{5}$ & 79.61 & 80.75 & 79.07 & 77.79 & 78.62 & 82.47 \\
$\mathbf{1 0}$ & 57.61 & 57.83 & 57.91 & 57.14 & 57.57 & 59.62 \\
$\mathbf{1 5}$ & 41.98 & 42.29 & 43.15 & 42.19 & 42.43 & 43.15 \\
$\mathbf{2 0}$ & 30.92 & 30.94 & 31.8 & 31.47 & 31.51 & 31.56 \\
\hline \hline
\end{tabular}

Table 3. Relative depth doses (percentage) calculated by different dose calculation algorithms and 2 Monte Carlo codes (MCNPX and GATE) for $1.5 \times 1.5 \mathrm{~cm}^{2}$ field size.

\begin{tabular}{ccccccc}
\hline \hline $\begin{array}{c}\text { Depth } \\
(\mathbf{c m})\end{array}$ & $\begin{array}{c}\text { MCNPX } \\
(\boldsymbol{\%})\end{array}$ & $\begin{array}{c}\text { GATE } \\
(\boldsymbol{\%})\end{array}$ & $\begin{array}{c}\text { CCC } \\
(\boldsymbol{\%})\end{array}$ & $\begin{array}{c}\text { Superposition } \\
(\boldsymbol{\%})\end{array}$ & $\begin{array}{c}\text { FFT } \\
(\boldsymbol{\%})\end{array}$ & $\begin{array}{c}\text { Clarkson } \\
(\boldsymbol{\%})\end{array}$ \\
\hline $\mathbf{0}$ & 37.89 & 37.69 & 51.99 & 29.36 & 29.44 & 32.24 \\
$\mathbf{1 . 5}$ & 100 & 100 & 99.05 & 98.87 & 99.28 & 99.8 \\
$\mathbf{5}$ & 80.74 & 80.15 & 79.77 & 79.98 & 80.31 & 82.25 \\
$\mathbf{1 0}$ & 58.5 & 57.68 & 58.49 & 58.32 & 58.56 & 59.33 \\
$\mathbf{1 5}$ & 42.67 & 41.62 & 43.24 & 42.81 & 42.87 & 42.9 \\
$\mathbf{2 0}$ & 31.39 & 30.61 & 31.84 & 31.62 & 31.69 & 31.34 \\
\hline \hline
\end{tabular}




\section{Comparing dose profiles between TPS and MCNPX and Gate in the small field}

For comparing dose profiles in small fields, profiles in 2 depths were extracted: depth of $D_{\max }$ and $10 \mathrm{~cm}$ in a homogeneous water phantom. Profiles at $D_{\max }$ are presented in Figure 4. For $0.5 \times 0.5$ $\mathrm{cm}^{2}$ field, FWHMs were calculated 0.5266, 0.5161, 0.9672, $0.7385,0.7136$ and $0.5749 \mathrm{~cm}$ by MCNPX, GATE, CCC, superposition, FFT and Clarkson respectively. All discussed dose calculation algorithms overestimate FWHM against MCNPX or GATE. Differences were up to $0.4511 \mathrm{~cm}$. Errors in calculating FWHM against MCNPX were 83.6, 40.2, 35.5, and 9.2\% and against GATE were 87.4, 43.1, 38.3, and 11.4 for CC, superposition, FFT and Clarkson respectively. For $1 \times 1 \mathrm{~cm}^{2}$ field, FWHMs were calculated 1.0581, 1.0438, 1.1548, 1.182, 1.1673 and $0.971 \mathrm{~cm}$ by MCNPX, GATE, CCC, superposition, FFT and Clarkson respectively. In this field, differences were up

(a)

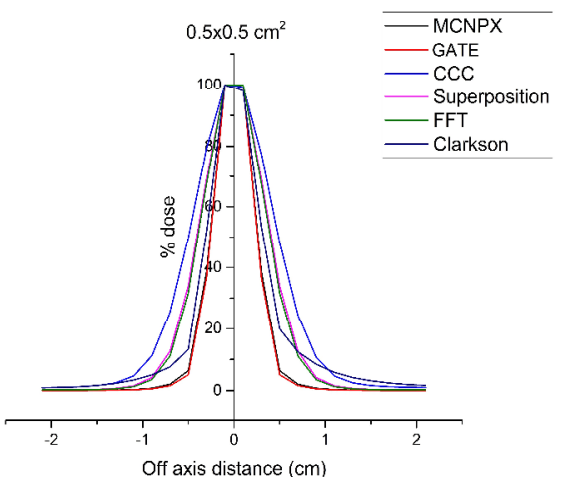

(b)

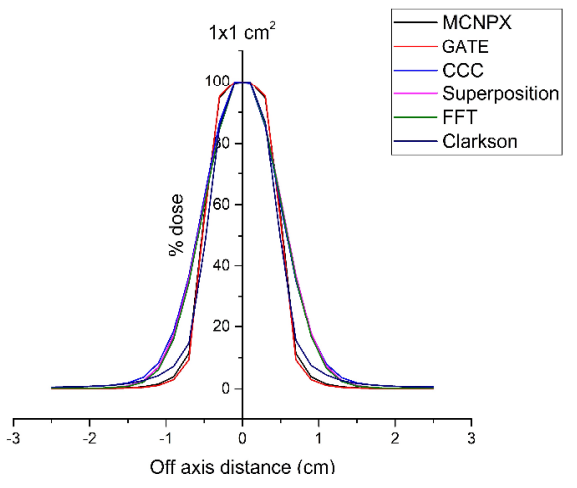

(c)

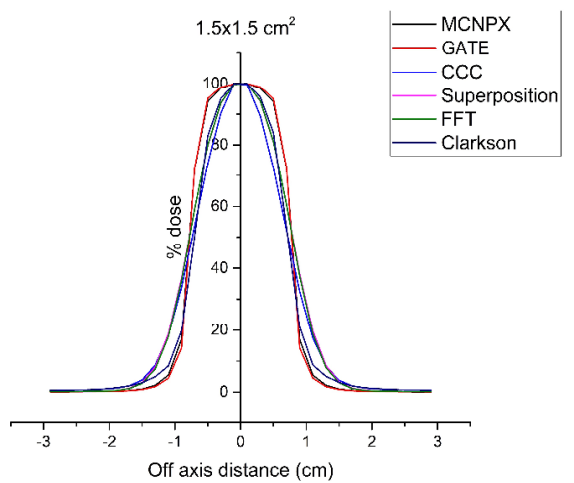

Figure 4. Lateral dose profiles for small fields in a homogenous water phantom at depth $1.5 \mathrm{~cm}$ across beam's central axis, a comparison between MCNPX, GATE and various dose calculation algorithms in different field sizes. A) $0.5 \times 0.5 \mathrm{~cm}^{2}$, B) $1 \times 1 \mathrm{~cm}^{2}$ and C) $1.5 \times 1.5 \mathrm{~cm}^{2}$. to $0.1382 \mathrm{~cm}$. Differences with MCNPX were $9.4 \% 11.7 \%$, $10.3 \%$ and $-8.2 \%$ and with GATE were $10.6 \%, 13.2 \%, 11.8 \%$, and $-7 \%$ for CCC, superposition, FFT and Clarkson respectively. For the $1.5 \times 1.5 \mathrm{~cm}^{2}$ field, FWHM was calculated $1.5598,1.5532,1.4431,1.576,1.5686$ and $1.4198 \mathrm{~cm}$ by MCNPX, GATE, CCC, superposition, FFT and Clarkson respectively. In this field, differences were up to $-0.1334 \mathrm{~cm}$ which is for the Clarkson algorithm. Differences in FWHM with MCNPX were $-7.5 \%, 1 \%, 0.6 \%$ and $-9 \%$ and with GATE were $-7.1 \%, 1.5 \%, 1 \%$ and $-8.6 \%$ for CCC, superposition, FFT and Clarkson respectively. Profiles for depth $10 \mathrm{~cm}$ through homogeneous water phantom are presented in Figure 5. In this depth, there were some differences in FWHM calculated by dose calculation algorithms and Monte Carlo simulation. Calculated FWHM for different algorithms and field sizes are presented in Table 4.

(a)

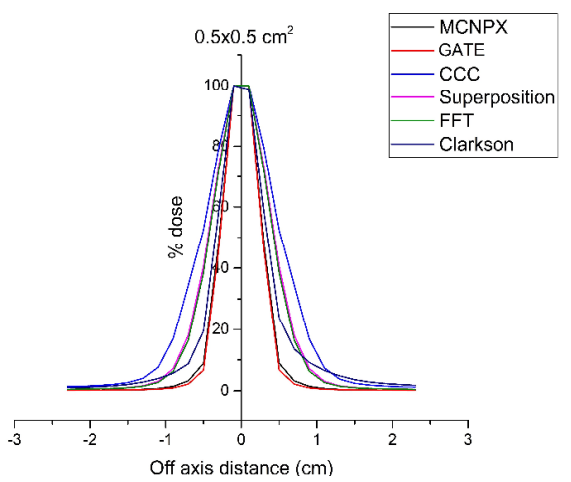

(b)

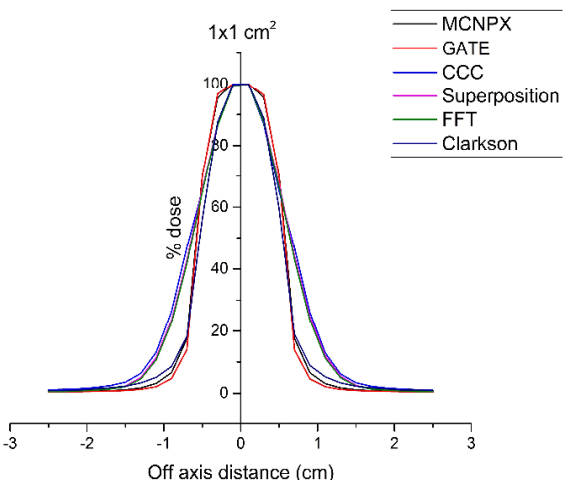

(c)



Figures5. Lateral dose profiles for small fields in a homogenous water phantom at depth $10 \mathrm{~cm}$ across beam's central axis, a comparison between MCNPX, GATE and various dose calculation algorithms in different field sizes. A) $\left.0.5 \times 0.5 \mathrm{~cm}^{2}, \mathrm{~B}\right) 1 \times 1 \mathrm{~cm}^{2}$ and C) $1.5 \times 1.5 \mathrm{~cm}^{2}$. 
Table 4. FWHM values (in $\mathrm{cm}$ ) for different dose calculation algorithms in various field sizes and depths.

\begin{tabular}{|c|c|c|c|c|c|c|c|}
\hline Field size & Depth $(\mathrm{cm})$ & MCNPX (cm) & GATE (cm) & $\mathrm{CCC}(\mathrm{cm})$ & Superposition $(\mathrm{cm})$ & FFT $(\mathbf{c m})$ & Clarkson $(\mathrm{cm})$ \\
\hline \multirow{2}{*}{$0.5 \times 0.5 \mathrm{~cm}^{2}$} & 1.5 & 0.527 & 0.516 & 0.967 & 0.738 & 0.713 & 0.575 \\
\hline & 10 & 0.5848 & 0.568 & 1.026 & 0.81 & 0.7801 & 0.6147 \\
\hline \multirow{2}{*}{$1 \times 1 \mathrm{~cm}^{2}$} & 1.5 & 1.0581 & 1.043 & 1.154 & 1.182 & 1.167 & 0.971 \\
\hline & 10 & 1.153 & 1.1415 & 1.326 & 1.2845 & 1.2677 & 1.0782 \\
\hline \multirow{2}{*}{$1.5 \times 1.5 \mathrm{~cm}^{2}$} & 1.5 & 1.5598 & 1.5532 & 1.4431 & 1.576 & 1.5686 & 1.4198 \\
\hline & 10 & 1.66 & 1.6472 & 1.643 & 1.7159 & 1.7079 & 1.5563 \\
\hline
\end{tabular}

\section{Discussion}

The use of modern radiation therapy techniques such as IMRT, VMAT, SRS, SBRT, and IGRT had led to superior accuracy in dose delivery. These techniques use small beamlets in treatment delivery and employ complex dose calculation algorithms in treatment planning systems. In IMRT and VMAT, intensity modulation is achieved using small fields produced by multileaf collimators (MLC) routinely. In the VMAT technique, gantry rotation and dose rate also are free degrees and its complexity is more than IMRT. So the requirement for dose verification of small fields is more important.

Small fields have a high dose gradient that delivers a high dose to the tumor and spare surrounding normal tissues. So determining dose in small beams is highly desired, but because of loss of lateral charged particle equilibrium, partial occlusion of primary photons from source and size of detectors, dosimetry in the small field is difficult. Therefore, it is important to investigate their effect on dose calculation algorithms used in treatment planning systems. Various publications have discussed and investigated the accuracy of different dose calculation algorithms against MC simulations or direct measurement with different dosimeters for small field sizes. $1,2,6,18,27,28,31-33,37-39$ The goal of this article was to investigate the accuracy of CCC, superposition, FFT and Clarkson dose calculation algorithms in small photon fields against two Monte Carlo codes: MCNPX and GATE. Validations of two models were carried out by using 3 fields $\left(6 \times 6,10 \times 10\right.$ and $\left.20 \times 20 \mathrm{~cm}^{2}\right)$. PDD results showed a good agreement between MC simulations and measurements by the ionization chamber (under $2.5 \%$ difference for all fields and codes). For the buildup region, a local differences of up to $15 \%$ for all fields and codes were observed. These differences also reported in other publications ${ }^{2}$ and reported to be because of reading uncertainties in the ionization chamber. ${ }^{2,22}$ Also it might be for volume averaging in dosimeter because of the high dose gradient in this region. ${ }^{17}$ For lateral dose profiles, our results for $6 \times 6,10 \times 10$ and $20 \times 20 \mathrm{~cm}^{2}$ fields meet with the criteria purposed by Venselaar et al. ${ }^{36}$ and Mesbahi et al. ${ }^{22}$ for MC codes very well.

As mentioned there are 2 types of dose calculation algorithms that are used in the treatment planning systems. Between the four algorithms used in this study, Clarkson is measurement-based, CCC, superposition, and FFT are model-based. We investigate the accuracy of these algorithms in a homogeneous water phantom against MC simulation. For Clarkson algorithm differences up to $7.5 \%$ were observed in PDDs, smaller discrepancies were observed for larger fields. CC algorithm overestimated dose in PDDs against MC simulations for all fields up to $2.5 \%$ which decreased with the increase of field sizes. Superposition and FFT algorithm showed similar behaviors, they both overestimated dose in PDD curves which differences are maximum for smaller field size. Similar results were reported in various publications for different types of algorithms. The FWHMs obtained from discussed algorithms had more differences in smaller fields, and CCC had the most difference in $0.5 \times 0.5 \mathrm{~cm}^{2}$. These differences showed the inability of these algorithms to calculate dose profiles in so small fields. When the field size increased, the differences in the FWHM decreased dramatically. This is in an agreement with previous studies. ${ }^{1}$ Behinaein et al investigated the accuracy of $\mathrm{AAA}$ and $\mathrm{AXB}$ algorithms against measurement with plastic scintillator and Monte Carlo simulation both inhomogeneous and heterogeneous situations for small photon fields. ${ }^{1}$ They used PDD and Profiles to compare algorithms. In homogeneous water phantom, they observed both algorithms agreed well with MC simulation and measurement with the plastic scintillator. Also, they reported that in a heterogeneous situation both algorithms have a good dose prediction comparing to MC simulation and measurement, although they mentioned a superior accuracy for the AXB algorithm. Chopra et al evaluated the accuracy of 5 dose calculation algorithms used in 3 treatment planning systems in the presence of tissue heterogeneous for a range of field sizes, from small to large. ${ }^{40}$ They used the ion chamber and EDR film for PDD and dose profile measurements in a range of field sizes. They observed that an MC algorithm used in Brainlab TPS has a good agreement compared to measurements (less than $3 \%$ ), and agreement improved with the increase of field size. Although the PB algorithm in this TPS significantly over predicted dose against the measurement (up to 34\%). Their results showed differences between $\mathrm{PB}$ and measurements which increased with the decrease of field size, decreasing phantom density and increasing depth within heterogeneity. AAA algorithm used in Eclipse TPS, over predicted dose compare to measurements, on the other hand, the AXB algorithm showed a good agreement with measurement for heterogeneous media as well as for interface region. Ojala et al evaluated the accuracy of 3 dose calculation algorithms, PBC, $\mathrm{AAA}$, and $\mathrm{AXB}$ which were used in the Eclipse treatment planning system for several small tumors placed in lung patients. ${ }^{6}$ They used dose distribution from a full Monte Carlo 
simulation as a reference. AXB showed a good agreement with MC simulation but for smaller PTVs, larger discrepancies observed. For AAA and PBC mean dose to smaller sized PTVs had discrepancies up to $20 \%$ and $60 \%$ respectively. Stathakis et al investigated the accuracy of the AXB algorithm against AAA, $\mathrm{CCC}$, and $\mathrm{MC}$ simulation in a homogeneous and heterogeneous medium for a set of small fields. ${ }^{41}$ Their results showed good agreement for all algorithms in homogeneous phantom which differences were $\pm 1.5 \%$. For Heterogeneous phantom, AXB and CCC showed a good agreement with MC simulation (less than $2 \%$ difference) where for AAA differences were up to $5 \%$. Calvo et al evaluated the CCC algorithm (Pinnacle) for lesions with a diameter smaller than $3 \mathrm{~cm} .{ }^{37}$ Their results demonstrated a $5.6 \%$ difference in PTV dose between CCC and MC simulation. Fotina et al conducted a comparison between CCC (Master Plan) and MC simulation for SBRT and IMRT cases. ${ }^{39}$ The difference in PTV dose was reported 5\% among the CCC dose calculation algorithm and MC simulation.

As declared our results for measurement-based and modelbased algorithms were in an agreement with previous studies. For the Clarkson algorithm, our results showed a much smaller deviation with MC simulation than previous publications, ${ }^{\mathbf{6} 40}$ which is because of this fact that those studies evaluated a measurement-based algorithm in a heterogeneous situation (mostly lung case study) but in this study, such an algorithm was evaluated in a homogeneous water phantom. Model-based algorithms in this study showed an acceptable agreement with MC simulation (less than 5\% difference). CCC algorithm had a very large difference in the buildup region which is due to the fact that this algorithm does not model the buildup region when entering from a low density to a high-density region. ${ }^{\mathbf{4 2}}$ All evaluated algorithms had better results in bigger fields and with a decrease of field size, deviations with MC simulation increased, as reported in previous studies. ${ }^{\mathbf{6 , 4 0 , 4 2}}$ The source of these differences in small fields is reported to be the loss of lateral charged particle equilibrium. ${ }^{\mathbf{4 2 , 4 3}}$ As reported by different authors most deviations appear in fields smaller than $1 \times 1 \mathrm{~cm}^{2}$. Palmans et al have stated that partial occlusion of primary photons from source happens when the field size is comparable with focal spot size, also they mentioned that in medical accelerators focal spot size is about $0.5 \mathrm{~cm} .{ }^{44}$ So in fields smaller than $1 \times 1 \mathrm{~cm}^{2}$, when the field size is comparable with focal spot size, partial occlusion of primary photons appears. AXB algorithm is reported to have superior accuracy against other model-based algorithms (like AAA and CCC) in various publications. , $\mathbf{6 , 4 0 , 4 1}$ Model-based algorithms use precalculated dose kernels, while the AXB algorithm has a more detailed model and like MC uses direct models and solves physical interaction between particles. This algorithm accurately models primary photons from source and also scattered photon and electron fluence, for this job it solves the linear Boltzmann transform equation. ${ }^{\mathbf{4 2}}$

All model-based algorithms that investigated had an agreement with MC simulation in small photon fields in a homogenous media concerning this that CCC algorithm from Monaco TPS had a superior accuracy toward the others. Clarkson algorithm as we discussed as a correction based algorithm had good accuracy in larger fields and homogenous media, but with decrease of field size and appearing of loss of charged particle equilibrium its accuracy drops dramatically. For better evaluation of these algorithms for small fields, they must be challenged in a more complex situation like a heterogeneous media.

\section{Conclusion}

Different dosed calculation algorithms were evaluated and compared with MC simulation in small fields. Comparison is done with dosimetric parameters such as PDDs and dose profiles in a homogenous water phantom. Clarkson algorithm (measurement-based) showed a large discrepancy with MC simulation (up to 7.5\%). Model-based algorithms had better agreement with MC simulations. CCC dose calculation algorithm used in Monaco showed superior accuracy than superposition and FFT employed in Isogray TPS. For all algorithms, mean differences decreased with the increase of field size which is in an agreement with previous publications.

\section{Acknowledgements}

This article was derived from the M.Sc. thesis of Mojtaba Abazarfard in the Medical Physics Department at the Shahid Beheshti University of Medical Sciences. Also, the authors would like to appreciate the support from all staff of the radiotherapy center of Emam Hossein hospital.

\section{References}

1. Behinaein S, Osei E, Darko J, et al. Evaluating small field dosimetry with the Acuros XB (AXB) and analytical anisotropic algorithm (AAA) dose calculation algorithms in the eclipse treatment planning system. J Radiother Pract. 2019:1-12. https://doi.org/10.1017/S1460396919000104

2. Mesbahi A, Zergoug I. Dose calculations for lung inhomogeneity in high-energy photon beams and small beamlets: a comparison between XiO and TiGRT treatment planning systems and MCNPX Monte Carlo code. Iran J Med Phys. 2015;12(3):167-77. https://doi.org/10.22038/IJMP.2015.6218

3. Alfonso R, Andreo P, Capote R, et al. A new formalism for reference dosimetry of small and nonstandard fields. Med Phys. 2008;35(11):5179-86. https://doi.org/10.1118/1.3005481 
4. Park JC, Li JG, Arhjoul L, et al. Adaptive beamlet-based finite-size pencil beam dose calculation for independent verification of IMRT and VMAT. Med Phys. 2015;42(4):1836-1850. doi:10.1118/1.4914858

5. Khan FM, Gibbons JP. Khan's the physics of radiation therapy: Lippincott Williams \& Wilkins; 2014.

6. Ojala JJ, Kapanen MK, Hyödynmaa SJ, et al. Performance of dose calculation algorithms from three generations in lung SBRT: comparison with full Monte Carlo-based dose distributions. J Appl Clin Med Phys. 2014;15(2):4-18. https://doi.org/10.1120/jacmp.v15i2.4662

7. Lechner W, Wesolowska P, Azangwe G, et al. A multinational audit of small field output factors calculated by treatment planning systems used in radiotherapy. PhiRO. 2018;5:58-63. https://doi.org/10.1016/j.phro.2018.02.005

8. Azangwe G, Grochowska P, Georg D, et al. Detector to detector corrections: a comprehensive experimental study of detector specific correction factors for beam output measurements for small radiotherapy beams. Med Phys. 2014;41(7):072103. https://doi.org/10.1118/1.4883795

9. Das IJ, Ding GX, Ahnesjö A. Small fields: nonequilibrium radiation dosimetry. Med Phys. 2008;35(1):206-15. https://doi.org/10.1118/1.2815356

10. Westermark M, Arndt J, Nilsson B, et al. Comparative dosimetry in narrow high-energy photon beams. Phys Med Biol. 2000;45(3):685. https://doi.org/10.1088/0031-9155/45/3/308

11. Scott AJ, Nahum AE, Fenwick JD. Using a Monte Carlo model to predict dosimetric properties of small radiotherapy photon fields. Med Phys. 2008;35(10):4671-84. https://doi.org/10.1118/1.2975223

12. Gholami S, Longo F, Nedaie HA, et al. Application of Geant4 Monte Carlo simulation in dose calculations for small radiosurgical fields. Med Dosim. 2018;43(3):214-223. https://doi.org/10.1016/j.meddos.2017.08.007

13. Partanen M, Ojala J, Niemelä J, et al. Comparison of two Monte Carlo-based codes for small-field dose calculations in external beam radiotherapy. Acta Oncol. 2017;56(6):891-3. https://doi.org/10.1080/0284186X.2017.1292048

14. Cranmer-Sargison G. Small field dosimetry: experimental methods and monte carlo simulation in small field radiation therapy dosimetry [Ph.D. thesis]. University of Leeds (United Kingdom); 2014.

15. Sterpin E, Tomsej M, De Smedt B, et al. Monte Carlo evaluation of the AAA treatment planning algorithm in a heterogeneous multilayer phantom and IMRT clinical treatments for an Elekta SL25 linear accelerator. Med Phys. 2007;34(5):1665-77. https://doi.org/10.1118/1.2727314

16. Mostaar A, Allahverdi M, Shahriari M. Application of MCNP4C Monte Carlo code in radiation dosimetry in heterogeneous phantom. Int J Radiat Res. 2003;1(3):143-149.

17. Mesbahi A, Fix M, Allahverdi M, et al. Monte Carlo calculation of Varian 2300C/D Linac photon beam characteristics: a comparison between MCNP4C, GEANT3 and measurements. Appl Radiat Isotopes. 2005;62(3):469-77. https://doi.org/10.1016/j.apradiso.2004.07.008

18. Gagné IM, Zavgorodni S. Evaluation of the analytical anisotropic algorithm in an extreme water-lung interface phantom using Monte Carlo dose calculations. J Appl Clin Med Phys. 2007;8(1):33-46. https://doi.org/10.1120/jacmp.v8i1.2324

19. Elcim Y, Dirican B, Yavas O. Dosimetric comparison of pencil beam and Monte Carlo algorithms in conformal lung radiotherapy. $\mathrm{J}$ Appl Clin Med Phys. 2018;19(5):616-24. https://doi.org/10.1002/acm2.12426

20. Hoskin P. External Beam Therapy: Oxford University Press; 2019.

21. Verhaegen F, Seuntjens J. Monte Carlo modelling of external radiotherapy photon beams. Phys Med Biol. 2003;48(21):R107. https://doi.org/10.1088/0031-9155/48/21/R01

22. Mesbahi A, Reilly AJ, Thwaites DI. Development and commissioning of a Monte Carlo photon beam model for Varian Clinac 2100EX linear accelerator. Appl Radiat Isotopes. 2006;64(6):656-62. https://doi.org/10.1016/j.apradiso.2005.12.012

23. Jan S, Benoit D, Becheva E, et al. GATE V6: a major enhancement of the GATE simulation platform enabling modelling of CT and radiotherapy. Phys Med Biol. 2011;56(4):881. https://doi.org/10.1088/0031-9155/56/4/001

24. Sarrut D, Bardiès M, Boussion N, et al. A review of the use and potential of the GATE Monte Carlo simulation code for radiation therapy and dosimetry applications. Med Phys. 2014;41(6Part1). https://doi.org/10.1118/1.4871617

25. Palta JR, Liu C, Li JG. Quality assurance of intensity-modulated radiation therapy. Int J Radiat Oncol. Biol. Phys.. 2008;71(1):S108S12. https://doi.org/10.1016/j.ijrobp.2007.05.092

26. Benedict SH, Yenice KM, Followill D, et al. Stereotactic body radiation therapy: the report of AAPM Task Group 101. Med Phys. 2010;37(8):4078-101. https://doi.org/10.1118/1.3438081

27. Fogliata A, Lobefalo F, Reggiori G, et al. Evaluation of the dose calculation accuracy for small fields defined by jaw or MLC for AAA and Acuros XB algorithms. Med Phys. 2016;43(10):5685-94. https://doi.org/10.1118/1.4963219

28. Fogliata A, Cozzi L. Dose calculation algorithm accuracy for small fields in non-homogeneous media: the lung SBRT case. Phys Medica. 2017;44:157-62. https://doi.org/10.1016/j.ejmp.2016.11.104 
29. Cranmer-Sargison G, Beckham W, Popescu I. Modelling an extreme water-lung interface using a single pencil beam algorithm and the Monte Carlo method. Phys Med Biol. 2004;49(8):1557. https://doi.org/10.1088/0031-9155/49/8/013

30. Krieger T, Sauer OA. Monte Carlo-versus pencil-beam-/collapsed-cone-dose calculation in a heterogeneous multi-layer phantom. Phys Med Biol. 2005;50(5):859. https://doi.org/10.1088/0031-9155/50/5/010

31. Fogliata A, Nicolini G, Clivio A, et al. Accuracy of Acuros XB and AAA dose calculation for small fields with reference to RapidArc $\mathbb{B}$ stereotactic treatments. Med Phys. 2011;38(11):6228-37. https://doi.org/10.1118/1.3654739

32. Huang B, Wu L, Lin P, et al. Dose calculation of Acuros XB and Anisotropic Analytical Algorithm in lung stereotactic body radiotherapy treatment with flattening filter free beams and the potential role of calculation grid size. Radiat Oncol. 2015;10(1):53. https://doi.org/10.1186/s13014-015-0357-0

33. Fogliata A, Nicolini G, Clivio A, et al. Dosimetric evaluation of Acuros XB Advanced Dose Calculation algorithm in heterogeneous media. Radiat Oncol. 2011;6(1):82. https://doi.org/10.1186/1748-717X-6-82

34. Pelowitz DB. MCNPX user's manual version 2.5. 0. Los Alamos National Laboratory. 2005;76:473.

35. Mesbahi A. Dosimetric characteristics of unflattened $6 \mathrm{MV}$ photon beams of a clinical linear accelerator: a Monte Carlo study. Appl Radiat Isotopes. 2007;65(9):1029-36. https://doi.org/10.1016/j.apradiso.2007.04.023

36. Venselaar J, Welleweerd H, Mijnheer B. Tolerances for the accuracy of photon beam dose calculations of treatment planning systems. Radiother Oncol. 2001;60(2):191-201. https://doi.org/10.1016/S0167-8140(01)00377-2

37. Calvo OI, Gutiérrez AN, Stathakis $\mathrm{S}$, et al. On the quantification of the dosimetric accuracy of collapsed cone convolution superposition (CCCS) algorithm for small lung volumes using IMRT. J Appl Clin Med Phys. 2012;13(3):43-59. https://doi.org/10.1120/jacmp.v13i3.3751

38. Carrasco P, Jornet N, Duch MA, et al. Comparison of dose calculation algorithms in phantoms with lung equivalent heterogeneities under conditions of lateral electronic disequilibrium: dose calculation algorithms in lung heterogeneities. Med Phys. 2004;31(10):2899911. https://doi.org/10.1118/1.1788932

39. Fotina I, Kragl G, Kroupa B, et al. Clinical comparison of dose calculation using the enhanced collapsed cone algorithm vs. a new Monte Carlo algorithm. Strahlenther Onkol. 2011;187(7):433-41. https://doi.org/10.1007/s00066-011-2215-9

40. Chopra KL, Leo P, Kabat C, et al. Evaluation of dose calculation accuracy of treatment planning systems in the presence of tissue heterogeneities. Ther Radiol Oncol. 2018;2:420-7. https://doi.org/10.21037/tro.2018.07.01

41. Stathakis S, Esquivel C, Quino LV, et al. Accuracy of the small field dosimetry using the Acuros XB dose calculation algorithm within and beyond heterogeneous media for $6 \mathrm{MV}$ photon beams. Int J Med Phys Clin Eng Radiat Oncol. 2012 ; 1 : $78-87$. https://doi.org/10.4236/ijmpcero.2012.13011

42. Najafzadeh M, Nickfarjam A, Jabbari K, et al. Dosimetric verification of lung phantom calculated by collapsed cone convolution: A Monte Carlo and experimental evaluation. J X-Ray Sci Technol. 2019;27(1):161-75. https://doi.org/10.3233/XST-180425

43. Caccia B, Andenna C, Iaccarino G, et al. Monte Carlo as a tool to evaluate the effect of different lung densities on radiotherapy dose distribution. Radiat Prot Dosim. 2014;162(1-2):115-9. https://doi.org/10.1093/rpd/ncu241

44. Palmans H, Andreo P, Huq MS, et al. Dosimetry of small static fields used in external photon beam radiotherapy: Summary of TRS483, the IAEA-AAPM international Code of Practice for reference and relative dose determination. Med Phys. 2018;45(11):e1123e45. https://doi.org/10.1002/mp.13208 